\title{
On Levinson's operator inequality and its converses
}

\author{
Jadranka Mićić ${ }^{*}$ and Josip Pečarić ${ }^{2}$
}

\author{
"Correspondence: jmicic@fsb.hr \\ ${ }^{1}$ Faculty of Mechanical Engineering \\ and Naval Architecture, University of \\ Zagreb, Ivana Lučića 5, Zagreb, \\ 10000, Croatia \\ Full list of author information is \\ available at the end of the article
}

\begin{abstract}
We give new results on Levinson's operator inequality and its converse for normalized positive linear mappings and some large class of ' 3 -convex functions at a point $c$ '.
\end{abstract}

MSC: Primary 47A63; secondary 47B15

Keywords: self-adjoint operator; positive linear mapping; convex function; Levinson's inequality; Mond-Pečarić method

\section{Introduction and preliminary results}

Let $\mathcal{B}(H)$ be the algebra of all bounded linear operators on a complex Hilbert space $H$. We denote by $\mathcal{B}_{h}(H)$ the real subspace of all self-adjoint operators on $H$. Bounds of $X \in \mathcal{B}_{h}(H)$ are defined by $m:=\inf \{\langle X \xi, \xi\rangle: \xi \in H,\|\xi\|=1\}$ and $M:=\sup \{\langle X \xi, \xi\rangle: \xi \in H,\|\xi\|=1\}$.

A continuous real valued function $f$ defined on an interval $I$ is said to be operator convex if $f(\lambda X+(1-\lambda) Y) \leq \lambda f(X)+(1-\lambda) f(Y)$ for all self-adjoint operators $X, Y$ with spectra contained in $I$ and all $\lambda \in[0,1]$. If the function $f$ is operator convex, then the so-called Jensen operator inequality $f(\Phi(X)) \leq \Phi(f(X))$ holds for any unital positive linear mapping $\Phi$ on $\mathcal{B}(H)$ and any $X \in \mathcal{B}_{h}(H)$ with spectrum contained in $I$. Many other versions of Jensen's operator inequality can be found in $[1,2]$.

Assume furthermore that $\left(\Phi_{1}, \ldots, \Phi_{n}\right)$ is an $n$-tuple of positive linear mappings $\Phi_{i}$ : $\mathcal{B}(H) \rightarrow \mathcal{B}(K)$. If in addition $\sum_{i=1}^{n} \Phi_{i}\left(1_{H}\right)=1_{K}$, we say that $\sum_{i=1}^{n} \Phi_{i}\left(1_{H}\right)=1_{K}$ is unital.

Now we give the definition of classes of functions for which we observe Levinson's operator inequality.

Definition 1 ([3]) Let $f \in \mathcal{C}(I)$ be a real valued function on an arbitrary interval $I$ in $\mathbb{R}$ and $c \in I^{\circ}$, where $I^{\circ}$ is the interior of $I$.

We say that $f \in \mathcal{K}_{1}^{c}(I)$ (resp. $f \in \mathcal{K}_{2}^{c}(I)$ ) if there exists a constant $\alpha$ such that the function $F(t)=f(t)-\frac{\alpha}{2} t^{2}$ is concave (resp. convex) on $I \cap(-\infty, c]$ and convex (resp. concave) on $I \cap[c, \infty)$. (See Figure 1.)

Moreover, we say that $f \in \dot{\mathcal{K}}_{1}^{c}(I)\left(\right.$ resp. $\left.f \in \dot{\mathcal{K}}_{2}^{c}(I)\right)$ if there exists a constant $\alpha$ such that the function $F$ is operator concave (resp. operator convex) on $I \cap(-\infty, c]$ and operator convex (resp. operator concave) on $I \cap[c, \infty)$.

The class of functions $\mathcal{K}_{1}^{c}(I)$ can be interpreted as functions that are '3-convex at a point $c^{\prime}$ and extends 3-convex functions in the following sense: a function is 3 -convex on $I$ if and only if it is at every $c \in I^{\circ}$.

(C) 2015 Mićić and Pečarić; licensee Springer. This is an Open Access article distributed under the terms of the Creative Commons Attribution License (http://creativecommons.org/licenses/by/4.0), which permits unrestricted use, distribution, and reproduction in any medium, provided the original work is properly credited. 
Figure 1 Continous 3-convex function at a point $c$.

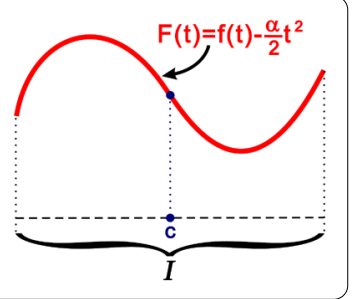

Next, we will review the history of research of Levison's inequality.

Levinson [4] considered an inequality as follows:

If $f:(0,2 c) \rightarrow \mathbb{R}$ satisfies $f^{\prime \prime \prime} \geq 0$ and $p_{i}, x_{i}, y_{i}, i=1,2, \ldots, n$, are such that $p_{i}>0$, $\sum_{i=1}^{n} p_{i}=1,0 \leq x_{i} \leq c$, and

$$
x_{1}+y_{1}=x_{2}+y_{2}=\cdots=x_{n}+y_{n}=2 c \text {, }
$$

then the inequality

$$
\sum_{i=1}^{n} p_{i} f\left(x_{i}\right)-f(\bar{x}) \leq \sum_{i=1}^{n} p_{i} f\left(y_{i}\right)-f(\bar{y})
$$

holds, where $\bar{x}=\sum_{i=1}^{n} p_{i} x_{i}$ and $\bar{y}=\sum_{i=1}^{n} p_{i} y_{i}$ denote the weighted arithmetic means.

Numerous papers have been devoted to generalizations and extensions of Levinson's result. Popoviciu [5] showed that the assumptions on the differentiability of $f$ can be weakened for (2); to hold it is enough to assume that $f$ is 3-convex. Bullen [6] gave another proof of Popoviciu's result rescaled to a general interval $[a, b]$.

Mercer [7] made a significant improvement by replacing (1) with the weaker condition that the variances of the two sequences are equal: $\sum_{i=1}^{n} p_{i}\left(x_{i}-\bar{x}\right)^{2}=\sum_{i=1}^{n} p_{i}\left(y_{i}-\bar{y}\right)^{2}$.

Witkowski $[8,9]$ extended this result in several ways. Firstly, he showed that Levinson's inequality can be stated in a more general setting with random variables. Furthermore, he showed that it is enough to assume that $f$ is 3 -convex and that the assumption of equality of the variances can be weakened to inequality in a certain direction.

Baloch et al. [10] built on and extended the methods of Witkowski [8]. They introduced a new class of functions $\mathcal{K}_{1}^{c}((a, b))$ as in Definition 1.

Mićić et al. [3] built on the methods given in [11] on operators. We give Levinson's operator inequality for unital fields of positive linear mappings and classes of functions given by Definition 1. Moreover, we considered order among quasi-arithmetic means under similar conditions.

Next, we give the main result in [3] for two operators and $f \in \dot{\mathcal{K}}_{i}^{c}(I), i=1,2$.

Theorem 1 Let $X, Y \in \mathcal{B}_{h}(H)$ be self-adjoint operators with spectra contained in $[m, M]$ and $[n, N]$, respectively, such that $a<m \leq M \leq c \leq n \leq N<b$. (See Figure 2.) Let $\Phi, \Psi$ be normalized positive linear mappings $\Phi, \Psi: \mathcal{B}(H) \rightarrow \mathcal{B}(K)$.

$$
\begin{aligned}
& \text { Iff } \in \dot{\mathcal{K}}_{1}^{c}((a, b)) \text { and } C_{1} \leq C_{2} \text {, then } \\
& \quad \Phi(f(X))-f(\Phi(X)) \leq C_{1} \leq C_{2} \leq \Psi(f(Y))-f(\Psi(Y)),
\end{aligned}
$$


Figure 2 Spectra conditions in Levinson's inequality for two operators and an operator 3-convex function.

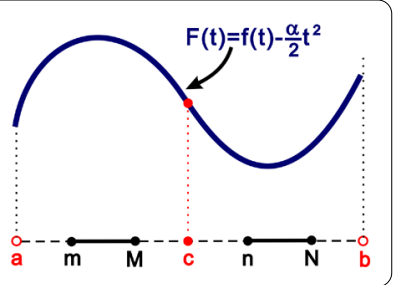

where

$$
C_{1}:=\frac{\alpha}{2}\left[\Phi\left(X^{2}\right)-\Phi(X)^{2}\right], \quad C_{2}:=\frac{\alpha}{2}\left[\Psi\left(Y^{2}\right)-\Psi(Y)^{2}\right] .
$$

But, iff $\in \dot{\mathcal{K}}_{2}^{c}((a, b))$ and $C_{1} \geq C_{2}$ holds, then the reverse inequalities are valid in (3).

Proof This theorem is special case of [3], Theorem 1, for $k=n=1$. For the sake of completeness, we give the proof.

Let $f \in \dot{\mathcal{K}}_{1}^{c}((a, b))$. So there is a constant $\alpha$ such that $F(t)=f(t)-\frac{\alpha}{2} t^{2}$ is operator concave on $[m, M] \subset(a, c]$. Jensen's inequality for an operator concave function implies

$$
0 \leq F(\Phi(X))-\Phi(F(X))=f(\Phi(X))-\frac{\alpha}{2} \Phi(X)^{2}-\Phi(f(X))+\frac{\alpha}{2} \Phi\left(X^{2}\right) .
$$

It follows that

$$
\Phi(f(X))-f(\Phi(X)) \leq C_{1}
$$

Similarly, since $F$ is operator convex on $[n, N] \subset[c, b)$, then Jensen's inequality for an operator convex function implies

$$
0 \leq \Psi(F(Y))-F(\Psi(Y))=\Psi(f(Y))-\frac{\alpha}{2} \Psi\left(Y^{2}\right)-f(\Psi(Y))+\frac{\alpha}{2} \Psi(Y)^{2} .
$$

It follows that

$$
C_{2} \leq \Psi(f(Y))-f(\Psi(Y))
$$

Combining inequalities (5) and (6) and taking into account that $C_{1} \leq C_{2}$ we obtain the desired inequality (3).

Applying Theorem 1 we obtain a version of Levinson's inequality with more operators as follows.

Corollary 2 ([3], Theorem 1$)$ Let $\left(X_{1}, \ldots, X_{k_{1}}\right)$ be a $k_{1}$-tuple and $\left(Y_{1}, \ldots, Y_{k_{2}}\right)$ be a $k_{2}$-tuple of self-adjoint operators $X_{i}, Y_{j} \in \mathcal{B}_{h}(H)$ with spectra contained in $[m, M]$ and $[n, N]$, respectively, such that $a<m \leq M \leq c \leq n \leq N<b$. Let $\left(\Phi_{1}, \ldots, \Phi_{k_{1}}\right)$ be a unital $k_{1}$-tuple and $\left(\Psi_{1}, \ldots, \Psi_{k_{2}}\right)$ be a unital $k_{2}$-tuple of positive linear mappings $\Phi_{i}, \Psi_{j}: \mathcal{B}(H) \rightarrow \mathcal{B}(K)$.

$$
\text { Iff } \in \dot{\mathcal{K}}_{1}^{c}((a, b)) \text { and } D_{1} \leq D_{2} \text {, then }
$$

$$
\sum_{i=1}^{k_{1}} \Phi_{i}\left(f\left(X_{i}\right)\right)-f\left(\sum_{i=1}^{k_{1}} \Phi_{i}\left(X_{i}\right)\right) \leq D_{1} \leq D_{2} \leq \sum_{i=1}^{k_{2}} \Psi_{i}\left(f\left(Y_{i}\right)\right)-f\left(\sum_{i=1}^{k_{2}} \Psi_{i}\left(Y_{i}\right)\right)
$$


holds, where

$$
\begin{aligned}
D_{1} & :=\frac{\alpha}{2}\left[\sum_{i=1}^{k_{1}} \Phi_{i}\left(X_{i}^{2}\right)-\left(\sum_{i=1}^{k_{1}} \Phi_{i}\left(X_{i}\right)\right)^{2}\right], \\
D_{2} & :=\frac{\alpha}{2}\left[\sum_{i=1}^{k_{2}} \Psi_{i}\left(Y_{i}^{2}\right)-\left(\sum_{i=1}^{k_{2}} \Psi_{i}\left(Y_{i}\right)\right)^{2}\right] . \\
\text { Iff } & \in \dot{\mathcal{K}}_{2}^{c}((a, b)) \text { and } D_{1} \geq D_{2} \text { holds, then the reverse inequalities are valid in (7). }
\end{aligned}
$$

Proof This result is proven directly in [3], Theorem 1, using Jensen's operator inequality on the sum of the operators. We will give the proof by applying Theorem 1 . We set $\tilde{X}=\operatorname{diag}\left(X_{1}, \ldots, X_{k_{1}}\right)$ and $\tilde{Y}=\operatorname{diag}\left(Y_{1}, \ldots, Y_{k_{2}}\right)$. Then $\tilde{X} \in \mathcal{B}_{h}(\underbrace{H \oplus \cdots \oplus H}_{k_{1}})$ and $\tilde{Y} \in$ $\mathcal{B}_{h}(\underbrace{H \oplus \cdots \oplus H}_{k_{2}})$, with spectra contained in $[m, M]$ and $[n, N]$, respectively. Also, we set $\tilde{\Phi}\left(\operatorname{diag}\left(A_{1}, \ldots, A_{k_{1}}\right)\right)=\sum_{i=1}^{k_{1}} \Phi_{i}\left(A_{i}\right)$ and $\tilde{\Psi}\left(\operatorname{diag}\left(B_{1}, \ldots, B_{k_{2}}\right)\right)=\sum_{i=1}^{k_{2}} \Psi_{i}\left(B_{i}\right)$. Then $\tilde{\Phi}$ : $\mathcal{B}(\underbrace{H \oplus \cdots \oplus H}_{k_{1}}) \rightarrow \mathcal{B}(K)$ and $\tilde{\Psi}: \mathcal{B}(\underbrace{H \oplus \cdots \oplus H}_{k_{2}}) \rightarrow \mathcal{B}(K)$ are normalized positive linear mappings. We have

$$
\tilde{C}_{1}=\frac{\alpha}{2}\left[\tilde{\Phi}\left(\tilde{X}^{2}\right)-\tilde{\Phi}(\tilde{X})^{2}\right]=\frac{\alpha}{2}\left[\sum_{i=1}^{k_{1}} \Phi_{i}\left(X_{i}^{2}\right)-\left(\sum_{i=1}^{k_{1}} \Phi_{i}\left(X_{i}\right)\right)^{2}\right]=D_{1}
$$

and

$$
\tilde{C}_{2}=\frac{\alpha}{2}\left[\tilde{\Psi}\left(\tilde{Y}^{2}\right)-\tilde{\Psi}(\tilde{Y})^{2}\right]=\frac{\alpha}{2}\left[\sum_{i=1}^{k_{2}} \Psi_{i}\left(Y_{i}^{2}\right)-\left(\sum_{i=1}^{k_{2}} \Phi_{i}\left(X_{i}\right)\right)^{2}\right]=D_{2} .
$$

Applying Theorem 1 on $\tilde{X}, \tilde{Y}, \tilde{\Phi}, \tilde{\Psi}$ and taking into account that $D_{1} \lesseqgtr D_{2}$ implies $\tilde{C}_{1} \lesseqgtr \tilde{C}_{2}$, we obtain the desired inequalities (7) or their reverse inequalities.

In this paper, as a continuation of the above consideration, we will observe other results as regards Levinson's operator inequality and its converse. We give a few examples for power functions.

\section{Converse of Levinson's operator inequality}

In this section we give the converse of inequalities (3) and (7) for $f \in \mathcal{K}_{i}^{c}(I), i=1,2$. First, for convenience we introduce some abbreviations.

Let $f:[m, M] \rightarrow \mathbb{R}, m<M$, such that $F(t)=f(t)-\frac{\alpha}{2} t^{2}, \alpha \in \mathbb{R}$, be a convex or a concave function. We denote a linear function through the points $(m, F(m))$ and $(M, F(M))$ by $f_{\alpha,[m, M]}^{\text {line }}$, i.e.

$$
f_{\alpha,[m, M]}^{\text {line }}(t)=\frac{M-t}{M-m} f(m)+\frac{t-m}{M-m} f(M)-\frac{\alpha}{2}((M+m) t-m M), \quad t \in \mathbb{R},
$$

and the slope of the line through $(m, F(m))$ and $(M, F(M))$ by $k_{\alpha, f[m, M]}$, i.e.

$$
k_{\alpha, f[m, M]}=\frac{f(M)-f(m)}{M-m}-\frac{\alpha}{2}(M+m) .
$$


Figure 3 Spectra conditions in the converse of Levinson's inequality for two operators and a 3-convex function.

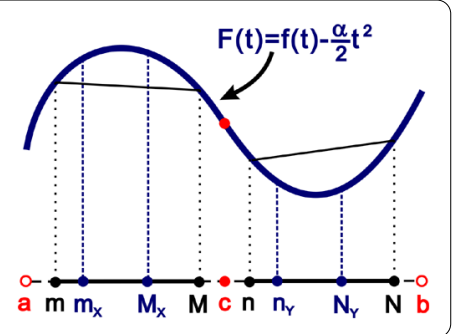

Next, we give the converse of Levinson's operator inequality for two operators.

Theorem 3 Let $X, Y, m, M, n, N, \Phi, \Psi, C_{1}, C_{2}$ be as in Theorem 1 and $m<M, n<N$. Let $m_{x}, M_{x}\left(m_{x} \leq M_{x}\right)$, and $n_{y}, N_{y}\left(n_{y} \leq N_{y}\right)$ be bounds of the operators $\Phi(X)$ and $\Psi(Y)$, respectively. (See Figure 3.)

Iff $\in \mathcal{K}_{1}^{c}((a, b))$ and $C_{1} \geq C_{2}$, then

$$
\Phi(f(X))-f(\Phi(X))+\beta_{1} 1_{K} \geq C_{1} \geq C_{2} \geq \Psi(f(Y))-f(\Psi(Y))+\beta_{2} 1_{K}
$$

where

$$
\begin{aligned}
& \beta_{1}=\max _{m_{x} \leq t \leq M_{x}}\left\{f(t)-\frac{\alpha}{2} t^{2}-f_{\alpha,[m, M]}^{\text {line }}(t)\right\} \geq 0, \\
& \beta_{2}=\min _{n_{y} \leq t \leq N_{y}}\left\{f(t)-\frac{\alpha}{2} t^{2}-f_{\alpha,[n, N]}^{\text {line }}(t)\right\} \leq 0 .
\end{aligned}
$$

The constants $\beta_{1}, \beta_{2}$ exist for any $\alpha, m, M, m_{x}, M_{x}$ and $n, N, n_{y}, N_{y}$.

The value of the constant $\beta_{1}$ is $\beta_{1}=f\left(t_{0}\right)-\frac{\alpha}{2} t_{0}^{2}-f_{\alpha,[m, M]}^{\text {line }}\left(t_{0}\right)$, where $t_{0}$ may be determined as follows:

- if $f_{-}^{\prime}(t)-\alpha t \leq k_{\alpha, f[m, M]}$ for every $t \in\left(m_{x}, M_{x}\right)$, then $t_{0}=m_{x}$,

- if $f_{-}^{\prime}\left(t_{1}\right)-\alpha t_{1} \geq k_{\alpha, f[m, M]} \geq f_{+}^{\prime}\left(t_{1}\right)-\alpha t_{1}$ for some $t_{1} \in\left(m_{x}, M_{x}\right)$, then $t_{0}=t_{1}$,

- if $f_{+}^{\prime}(t)-\alpha t \geq k_{\alpha, f[m, M]}$ for every $t \in\left(m_{x}, M_{x}\right)$, then $t_{0}=M_{x}$.

The value of $\beta_{2}$ can be determined as $\beta_{1}$ if we replace $m, M, m_{x}, M_{x}$ by $n, N, n_{y}, N_{y}$, respectively, and with reverse inequality signs.

In the dual case, if $f \in \mathcal{K}_{2}^{c}((a, b))$ and $C_{1} \leq C_{2}$ holds, then the reverse inequalities are valid in (9), where $\beta_{1} \leq 0$ with min instead of $\max$ in (10) and $\beta_{2} \geq 0$ with max instead of $\min$ in (11). The value of the constants $\beta_{1}$ and $\beta_{2}$ can be determined as above with reverse inequality signs.

Proof We will give the proof for $f \in \mathcal{K}_{1}^{c}((a, b))$. So there is a constant $\alpha$ such that $F(t)=$ $f(t)-\frac{\alpha}{2} t^{2}$ is concave on $[m, M] \subset(a, c]$. The converse of Jensen's operator inequality gives (see [12], Theorem 3.4)

$$
\begin{aligned}
& \Phi(F(X))-F(\Phi(X)) \geq \min _{m_{x} \leq t \leq M_{x}}\left\{f_{\alpha,[m, M]}^{\text {line }}(t)-f(t)-\frac{\alpha}{2} t^{2}\right\} 1_{K} \\
& \Rightarrow \quad \Phi(f(X))-\frac{\alpha}{2} \Phi\left(X^{2}\right)-f(\Phi(X))+\frac{\alpha}{2} \Phi(X)^{2}+\beta_{1} 1_{K} \geq 0 \\
& \Rightarrow \quad \Phi(f(X))-f(\Phi(X))+\beta_{1} 1_{K} \geq C_{1} .
\end{aligned}
$$


Similarly, since $F$ is operator convex on $[n, N] \subset[c, b)$, then Jensen's operator inequality gives (see [12], Theorem 3.4)

$$
\begin{aligned}
& \Psi(F(Y))-F(\Psi(Y)) \leq \max _{n_{y} \leq t \leq N_{y}}\left\{f_{\alpha,[n, N]}^{\text {line }}(t)-f(t)-\frac{\alpha}{2} t^{2}\right\} \\
& \quad \Rightarrow \quad \Psi(f(Y))-\frac{\alpha}{2} \Psi\left(Y^{2}\right)-f(\Psi(Y))+\frac{\alpha}{2} \Psi(Y)^{2}+\beta_{2} 1_{K} \leq 0 \\
& \Rightarrow \quad C_{2} \geq \Psi(f(Y))-f(\Psi(Y))+\beta_{2} 1_{K} .
\end{aligned}
$$

Combining inequalities (12) and (13) and taking into account $C_{1} \geq C_{2}$ we obtain the desired inequality (9). We obtain $\beta_{1}=f\left(t_{0}\right)-\frac{\alpha}{2} t_{0}^{2}-f_{\alpha,[m, M]}^{\text {line }}\left(t_{0}\right)$, where $t_{0}$ is determined as in the statement of Theorem 3, by applying [12], Theorem 3.4, to $\beta_{1}=-\min _{m_{x} \leq t \leq M_{x}}\left\{f_{\alpha,[m, M]}^{\text {line }}(t)-\right.$ $\left.f(t)+\frac{\alpha}{2} t^{2}\right\}$. Analogously we get $\beta_{2}=f\left(t_{0}\right)-\frac{\alpha}{2} t_{0}^{2}-f_{\alpha,[n, N]}^{\text {line }}\left(t_{0}\right)$.

Remark 1 Let the assumptions of Theorem 3 be satisfied. If $C_{1} \geq C_{2}, f$ is strictly concave differentiable on $[m, c]$ and strictly convex differentiable on $[c, N]$, then $(9)$ holds for

$$
\begin{aligned}
& \beta_{1}=f\left(x_{0}\right)-\frac{\alpha}{2} x_{0}^{2}-f_{\alpha,[m, M]}^{\text {line }}\left(x_{0}\right) \leq f\left(\bar{x}_{0}\right)-\frac{\alpha}{2} \bar{x}_{0}^{2}-f_{\alpha,[m, M]}^{\text {line }}\left(\bar{x}_{0}\right), \\
& \beta_{2}=f\left(y_{0}\right)-\frac{\alpha}{2} y_{0}^{2}-f_{\alpha,[n, N]}^{\text {line }}\left(y_{0}\right) \geq f\left(\bar{y}_{0}\right)-\frac{\alpha}{2} \bar{y}_{0}^{2}-f_{\alpha,[n, N]}^{\text {line }}\left(\bar{y}_{0}\right),
\end{aligned}
$$

where $x_{0}$ may be determined as follows:

- if $f^{\prime}\left(m_{x}\right)-\alpha m_{x} \leq k_{\alpha, f[m, M]}$, then $x_{0}=m_{x}$,

- if $f^{\prime}\left(m_{x}\right)-\alpha m_{x} \geq k_{\alpha, f[m, M]} \geq f^{\prime}\left(M_{x}\right)-\alpha M_{x}$, then $x_{0}$ is the unique solution of the equation $f^{\prime}(t)-\alpha t=k_{\alpha, f[m, M]}$,

- if $f^{\prime}\left(M_{x}\right)-\alpha M_{x} \geq k_{\alpha, f[m, M]}$, then $x_{0}=M_{x}$,

and $\bar{x}_{0}$ is the unique solution in $(m, M)$ of the equation $f^{\prime}(t)-\alpha t=k_{\alpha, f[m, M]}$.

The values of $y_{0}, \bar{y}_{0}$ can be determined as $x_{0}, \bar{x}_{0}$, if we replace $m, M, m_{x}, M_{x}$ by $n, N, n_{y}$, $N_{y}$, respectively, and with reverse inequality signs.

Example 1 Let $\Phi, \Psi, X, Y, m, M \geq 0, n, N \geq 0, m_{x}, M_{x}, n_{y}, N_{y}$ be as in Theorem 3 .

We will apply Theorem 3 putting $f(t)=t^{p}$ on $(0, c]$ and $f(t)=d t^{q}$ on $[c, \infty)$, where $c>0$ and $d=c^{p-q}$.

(i) If $p \in(-\infty, 0] \cup[1, \infty), q \in[0,1]$, and $\alpha=0$, then $f \in \mathcal{K}_{2}^{c}([0, \infty))$. So, (5) and the reverse of (9) give

$$
\Phi\left(X^{p}\right)-\Phi(X)^{p}+\beta_{1}^{\circ} 1_{K} \leq 0 \leq d \Psi\left(Y^{q}\right)-d \Psi(Y)^{q}+\alpha_{2} 1_{K} \leq \beta_{2}^{\circ} 1_{K},
$$

where

$$
\begin{aligned}
& \beta_{1}^{\circ}=\min _{m_{x} \leq t \leq M_{x}}\left\{t^{p}-\frac{M-t}{M-m} m^{p}-\frac{t-m}{M-m} M^{p}\right\} \leq 0, \\
& \beta_{2}^{\circ}=d \cdot \max _{n_{y} \leq t \leq N_{y}}\left\{t^{q}-\frac{N-t}{N-n} n^{q}-\frac{t-n}{N-n} N^{q}\right\} \geq 0 .
\end{aligned}
$$


(ii) If $p, q \in(-\infty, 0] \cup[1,2], p^{2}-p \geq q^{2}-q$, and $\alpha=c^{p-2}\left(p^{2}-p+q^{2}-q\right) / 2$, then

$$
\begin{aligned}
& \frac{d^{2}}{d t^{2}}\left(t^{p}-\frac{\alpha}{2} t^{2}\right)=p(p-1) t^{p-2}-\alpha \geq p(p-1) c^{p-2}-\alpha \geq 0, \quad \text { if } 0 \leq t \leq c \\
& \frac{d^{2}}{d t^{2}}\left(c^{p-q} t^{q}-\frac{\alpha}{2} t^{2}\right)=q(q-1) c^{p-q} t^{q-2}-\alpha \leq q(q-1) c^{p-2}-\alpha \leq 0, \quad \text { if } t \geq c .
\end{aligned}
$$

So, $f \in \mathcal{K}_{2}^{c}([0, \infty))$. If

$$
(0<) \quad C_{1}:=\frac{\alpha}{2}\left[\Phi\left(X^{2}\right)-\Phi(X)^{2}\right] \leq C_{2}:=\frac{\alpha}{2}\left[\Psi\left(Y^{2}\right)-\Psi(Y)^{2}\right]
$$

then the reverse of (9) gives

$$
\Phi\left(X^{p}\right)-\Phi(X)^{p}+\beta_{1} 1_{K} \leq C_{1} \leq C_{2} \leq d \Psi\left(Y^{q}\right)-d \Psi(Y)^{q}+\beta_{2} 1_{K}
$$

where

$$
\begin{aligned}
& \beta_{1}=\min _{m_{x} \leq t \leq M_{x}}\left\{t^{p}-\frac{M-t}{M-m} m^{p}-\frac{t-m}{M-m} M^{p}+\frac{\alpha}{2}\left((M+m) t-m M-t^{2}\right)\right\} \leq 0, \\
& \beta_{2}=\max _{n_{y} \leq t \leq N_{y}}\left\{d\left(t^{q}-\frac{N-t}{N-n} n^{q}-\frac{t-n}{N-n} N^{q}\right)+\frac{\alpha}{2}\left((N+n) t-n N-t^{2}\right)\right\} \geq 0 .
\end{aligned}
$$

(iii) If $p, q \in[0,1] \cup[2, \infty), p^{2}-p \leq q^{2}-q$, and $\alpha=c^{p-2}\left(p^{2}-p+q^{2}-q\right) / 2$, then

$$
\begin{aligned}
& \frac{d^{2}}{d t^{2}}\left(t^{p}-\frac{\alpha}{2} t^{2}\right) \leq p(p-1) c^{p-2}-\alpha \leq 0, \quad \text { if } 0 \leq t \leq c \\
& \frac{d^{2}}{d t^{2}}\left(c^{p-q} t^{q}-\frac{\alpha}{2} t^{2}\right) \geq q(q-1) c^{p-2}-\alpha \geq 0, \quad \text { if } t \geq c .
\end{aligned}
$$

So, $f \in \mathcal{K}_{1}^{c}([0, \infty))$. If $C_{1} \geq C_{2}$, then (9) gives

$$
\Phi\left(X^{p}\right)-\Phi(X)^{p}+\gamma_{1} 1_{K} \geq C_{1} \geq C_{2} \geq d \Psi\left(Y^{q}\right)-d \Psi(Y)^{q}+\gamma_{2} 1_{K}
$$

where $\gamma_{1} \geq 0$ is defined similar to $\beta_{1}$ with max instead of min and $\gamma_{2} \leq 0$ is defined similar to $\beta_{2}$ with min instead of max.

Remark 2 Let the assumptions of Theorem 3 be satisfied. If $f \in \dot{\mathcal{K}}_{1}^{c}((a, b))$ and $C_{1} \geq C_{2}$, we obtain the following extension of (9):

$$
\begin{aligned}
C_{1}+\beta_{1} 1_{K} & \geq \Phi(f(X))-f(\Phi(X))+\beta_{1} 1_{K} \geq C_{1} \geq C_{2} \\
& \geq \Psi(f(Y))-f(\Psi(Y))+\beta_{2} 1_{K} \geq C_{2}+\beta_{2} 1_{K} .
\end{aligned}
$$

In the dual case, if $f \in \dot{\mathcal{K}}_{2}^{c}((a, b))$ and $C_{1} \leq C_{2}$, then the reverse inequalities are valid in (14).

Applying Theorem 3 we obtain a version of the converse of Levinson's inequality with more operators. 
Corollary 4 Let $X_{i}, \Phi_{i}\left(i=1, \ldots, k_{1}\right), Y_{j}, \Psi_{j}\left(j=1, \ldots, k_{2}\right), m, M, n, N, D_{1}, D_{2}$ be as in Corollary 2. Let $m_{x}, M_{x}\left(m_{x} \leq M_{x}\right)$, and $n_{y}, N_{y}\left(n_{y} \leq N_{y}\right)$ be bounds of the operators $X=\sum_{i=1}^{k_{1}} \Phi_{i}\left(X_{i}\right)$ and $Y=\sum_{i=1}^{k_{2}} \Psi_{i}\left(Y_{i}\right)$, respectively. If $\in \mathcal{K}_{1}^{c}((a, b))$ and $D_{1} \geq D_{2}$, then

$$
\begin{aligned}
& \sum_{i=1}^{k_{1}} \Phi_{i}\left(f\left(X_{i}\right)\right)-f\left(\sum_{i=1}^{k_{1}} \Phi_{i}\left(X_{i}\right)\right)+\beta_{1} 1_{K} \\
& \quad \geq D_{1} \geq D_{2} \geq \sum_{i=1}^{k_{2}} \Psi_{i}\left(f\left(Y_{i}\right)\right)-f\left(\sum_{i=1}^{k_{2}} \Psi_{i}\left(Y_{i}\right)\right)+\beta_{2} 1_{K},
\end{aligned}
$$

where $\beta_{1}$ and $\beta_{2}$ are as in Theorem 3.

If $f \in \mathcal{K}_{2}^{c}((a, b))$ and $D_{1} \leq D_{2}$ holds, then the reverse inequalities are valid in (15) with $\beta_{1}$ and $\beta_{2}$ as in Theorem 3 in the dual case.

Proof We use the same technique as in the proof of Corollary 2. We omit the details.

Remark 3 Applying Corollary 4 to positive linear mappings $\Phi_{i}, \Psi: \mathcal{B}(H) \rightarrow \mathcal{B}(K)$ determined by $\Phi_{i}: B \mapsto p_{i} B, i=1, \ldots, k_{1}$, and $\Psi_{i}: B \mapsto q_{i} B, i=1, \ldots, k_{2}$, we obtain the following obvious result with convex combinations of the operators $X_{i}, i=1, \ldots, k_{1}$, and $Y_{j}$, $j=1, \ldots, k_{2}$ :

Let $X_{i}, Y_{j}$ be operators as in Corollary 4, such that $a<m_{x} \leq M_{x} \leq c \leq m_{y} \leq M_{y}<b$ for some $a, b, c \in \mathbb{R}$. Let $\left(p_{1}, \ldots, p_{k_{1}}\right)$ be a $k_{1}$-tuple and $\left(q_{1}, \ldots, q_{k_{2}}\right)$ be a $k_{2}$-tuple of positive scalars such that $\sum_{i=1}^{k_{1}} p_{i}=1$ and $\sum_{j=1}^{k_{2}} q_{j}=1$.

If $f \in \mathcal{K}_{1}^{c}((a, b))$ and $P \leq Q$, then

$$
\sum_{i=1}^{k_{1}} p_{i} f\left(X_{i}\right)-f(\bar{X})+\beta_{1} 1_{K} \leq P \leq Q \leq \sum_{j=1}^{k_{2}} q_{i} f\left(Y_{j}\right)-f(\bar{Y})+\beta_{2} 1_{K},
$$

where $\beta_{1}$ and $\beta_{2}$ are as in Theorem 3,

$$
P:=\frac{\alpha}{2} \sum_{i=1}^{k_{1}} p_{i}\left(X_{i}-\bar{X}\right)^{2}, \quad Q:=\frac{\alpha}{2} \sum_{j=1}^{k_{2}} q_{j}\left(Y_{j}-\bar{Y}\right)^{2},
$$

and $\bar{X}:=\sum_{i=1}^{k_{1}} p_{i} X_{i}, \bar{Y}:=\sum_{j=1}^{k_{1}} q_{j} Y_{j}$ denote the weighted arithmetic means of the operators.

\section{Refined Levinson's operator inequality}

In this section we obtain a refinement of Levison's operator inequality (7) given in Section 2 under weaker conditions.

The absolute value of $B \in \mathcal{B}(H)$ is defined by $|B|=\left(B^{*} B\right)^{1 / 2}$.

For convenience, we introduce the abbreviations $\bar{\Delta}$ and $\delta$ as follows:

$$
\text { - } \bar{\Delta} \equiv \bar{\Delta}_{B}(m, M):=\frac{1}{2} 1_{K}-\frac{1}{M-m}\left|B-\frac{m+M}{2} 1_{K}\right| \text {, }
$$

where $B \in \mathcal{B}_{h}(H)$ is a self-adjoint operator, $\Phi$ is a normalized positive linear mapping and $m, M(m<M)$ are some scalars such that spectra $\operatorname{Sp}(X) \subseteq[m, M]$. Since $m 1_{K} \leq B \leq M 1_{K}$, we have $-\frac{M-m}{2} 1_{K} \leq B-\frac{m+M}{2} 1_{K} \leq \frac{M-m}{2} 1_{K}$ and $0 \leq\left|\Phi(B)-\frac{m+M}{2} 1_{K}\right| \leq \frac{M-m}{2} 1_{K}$. It follows $\bar{\Delta} \geq 0$.

$$
\text { - } \delta \equiv \delta_{f, \alpha}(m, M):=2 f\left(\frac{m+M}{2}\right)-f(m)-f(M)+\frac{\alpha}{4}(M-m)^{2},
$$



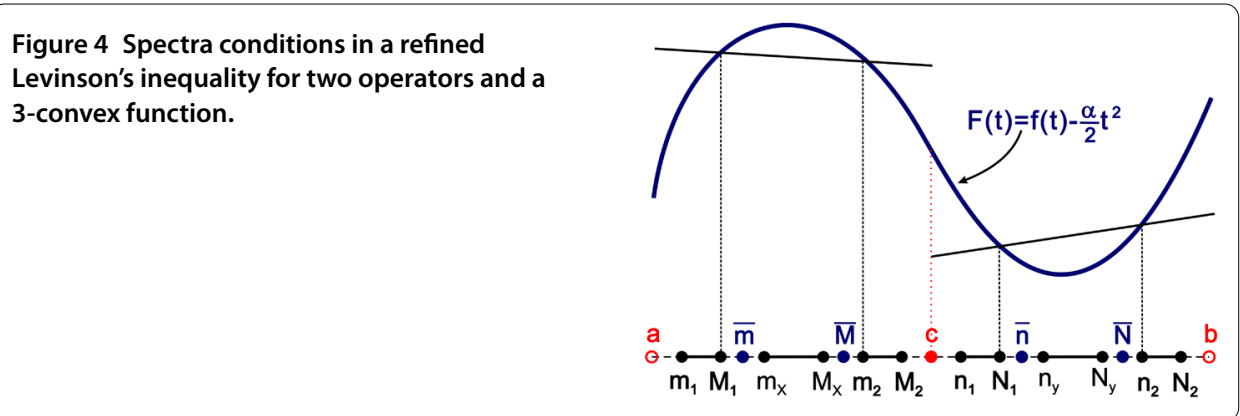

where $f:[m, M] \rightarrow \mathbb{R}$ is a continuous function and $\alpha \in \mathbb{R}$. Obviously, if $F(t)=f(t)-\frac{\alpha}{2} t^{2}$ is concave (resp. convex) then $\delta \geq 0$ (resp. $\delta \leq 0$ ).

First, we give refined Levinson's operator inequality for two pairs of operators.

Theorem 5 Let $\Phi, \Psi: \mathcal{B}(H) \oplus \mathcal{B}(H) \rightarrow \mathcal{B}(K)$ be normalized mappings such that $\Phi\left(\operatorname{diag}\left(B_{1}, B_{2}\right)\right)=\Phi_{1}\left(B_{1}\right)+\Phi_{2}\left(B_{2}\right)$ and $\Psi\left(\operatorname{diag}\left(B_{1}, B_{2}\right)\right)=\Psi_{1}\left(B_{1}\right)+\Psi_{2}\left(B_{2}\right)$, where $\Phi_{1}, \Phi_{2}$, $\Psi_{1}, \Psi_{2}$ are positive linear mappings.

Let $X=\operatorname{diag}\left(X_{1}, X_{2}\right), Y=\operatorname{diag}\left(Y_{1}, Y_{2}\right)$, where $X_{1}, X_{2}, Y_{1}, Y_{2} \in \mathcal{B}_{h}(H)$ are self-adjoint operators with spectra $\operatorname{Sp}\left(X_{1}\right) \subseteq\left[m_{1}, M_{1}\right], \operatorname{Sp}\left(X_{2}\right) \subseteq\left[m_{2}, M_{2}\right], \operatorname{Sp}\left(Y_{1}\right) \subseteq\left[n_{1}, N_{1}\right], \operatorname{Sp}\left(Y_{2}\right) \subseteq\left[n_{2}, N_{2}\right]$ $M_{1}<m_{2}, N_{1}<n_{2}$. Let $a<m_{1} \leq M_{1} \leq m_{x} \leq M_{x} \leq m_{2} \leq M_{2} \leq c \leq n_{1} \leq N_{1} \leq n_{y} \leq N_{y} \leq$ $n_{2} \leq N_{2}<b$, where $m_{x}, M_{x}$ and $n_{y}, N_{y}$ are bounds of $\Phi(X)$ and $\Psi(Y)$, respectively. (See Figure 4.)

Iff $\in \mathcal{K}_{1}^{c}((a, b))$ and $C_{1} \leq C_{2}($ see $(4))$, then

$$
\begin{aligned}
\Phi(f(X))-f(\Phi(X)) & \leq \Phi(f(X))-f(\Phi(X))+\delta_{1} \bar{X} \leq C_{1} \\
& \leq C_{2} \leq \Psi(f(Y))-f(\Psi(Y))+\delta_{2} \bar{Y} \leq \Psi(f(Y))-f(\Psi(Y)),
\end{aligned}
$$

where $\delta_{1}=\delta_{f, \alpha}(\bar{m}, \bar{M}) \geq 0, \bar{X}=\bar{\Delta}_{\Phi(X)}(\bar{m}, \bar{M}) \geq 0$ for arbitrary numbers $\bar{m} \in\left[M_{1}, m_{x}\right], \bar{M} \in$ $\left[M_{x}, m_{2}\right], \bar{m}<\bar{M}$ and $\delta_{2}=\delta_{f, \alpha}(\bar{n}, \bar{N}) \leq 0, \bar{Y}=\bar{\Delta}_{\Psi(Y)}(\bar{n}, \bar{N}) \geq 0$ for arbitrary numbers $\bar{n} \in$ $\left[N_{1}, n_{y}\right], \bar{N} \in\left[N_{y}, n_{2}\right], \bar{n}<\bar{N}$.

But, if $f \in \mathcal{K}_{2}^{c}((a, b))$ and $C_{1} \geq C_{2}$ holds, then the reverse inequalities are valid in (16), with $\delta_{1} \leq 0$ and $\delta_{2} \geq 0$.

Proof We will give the proof for $f \in \mathcal{K}_{1}^{c}((a, b))$. Since $F(t)=f(t)-\frac{\alpha}{2} t^{2}$ is concave on $\left[m_{1}, c\right] \subset$ $(a, c]$ for some constant $\alpha$, the refined Jensen's operator inequality for a concave function implies (see [13], Theorem 3)

$$
\begin{aligned}
& F(\Phi(X)) \geq \Phi(F(X))+\widetilde{\delta}_{1} \bar{X} \geq \Phi(F(X)) \\
& \quad \Rightarrow \quad C_{1} \geq \Phi(f(X))-f(\Phi(X))+\delta_{1} \bar{X} \geq \Phi(f(X))-f(\Phi(X)),
\end{aligned}
$$

since $0 \leq \widetilde{\delta}_{1}=2 F\left(\frac{\bar{m}+\bar{M}}{2}\right)-F(\bar{m})-F(\bar{M})=\delta_{f, \alpha}(\bar{m}, \bar{M})=\delta_{1}$ and

$$
\bar{X}=\frac{1}{2} 1_{K}-\frac{1}{\bar{M}-\bar{m}}\left|\Phi_{1}\left(X_{1}\right)+\Phi_{2}\left(X_{2}\right)-\frac{\bar{m}+\bar{M}}{2} 1_{K}\right|=\bar{\Delta}_{\Phi(X)}(\bar{m}, \bar{M}) .
$$


Similarly, since $F$ is convex on $\left[c, N_{2}\right] \subset[c, b)$ for some constant $\alpha$, the refined Jensen's operator inequality for a convex function implies (see [13], Theorem 3)

$$
\begin{aligned}
& F(\Psi(Y)) \leq \Psi(F(Y))-\widetilde{\delta}_{2} \bar{Y} \geq \Psi(F(Y)) \\
& \quad \Rightarrow \quad C_{2} \leq \Psi(f(Y))-f(\Psi(Y))+\delta_{2} \bar{Y} \leq \Psi(f(Y))-f(\Psi(Y)),
\end{aligned}
$$

since $0 \leq \widetilde{\delta}_{2}=F(\bar{N})+F(\bar{N})-2 F\left(\frac{\bar{n}+\bar{N}}{2}\right)=-\delta_{f, \alpha}(\bar{n}, \bar{N})=-\delta_{2}$ and

$$
\bar{Y}=\frac{1}{2} 1_{K}-\frac{1}{\bar{N}-\bar{n}}\left|\Psi_{1}\left(Y_{1}\right)+\Psi_{2}\left(Y_{2}\right)-\frac{\bar{n}+\bar{N}}{2} 1_{K}\right|=\bar{\Delta}_{\Psi(Y)}(\bar{n}, \bar{N}) .
$$

Combining inequalities (17) and (18) we obtain the desired inequality (16).

Example 2 Let $\Phi_{i}, \Psi_{i}, X_{i}, Y_{i}, m_{i}, M_{i} \geq 0, n_{i}, N_{i} \geq 0, i=1,2, \Phi, \Psi, X, Y, m_{x}, M_{x}, n_{y}, N_{y}$ be as in Theorem 5 .

We will use the same technique as in Example 1 and we will apply Theorem 5 putting $f(t)=t^{p}$ on $(0, c], f(t)=d t^{q}$ on $[c, \infty)$, where $c>0$ and $d=c^{p-q}$.

(i) If $p \in[0,1], q \in(-\infty, 0] \cup[1, \infty)$, and $\alpha=0$, then $f \in \mathcal{K}_{1}^{c}([0, \infty))$. So, (16) gives

$$
\Phi\left(X^{p}\right)-\Phi(X)^{p}+\delta_{1} \bar{X} \leq 0 \leq d \Psi\left(Y^{q}\right)-d \Psi(Y)^{q}+\delta_{2} \bar{Y}
$$

where

$$
\begin{aligned}
& \delta_{1}=2^{1-p}(\bar{m}+\bar{M})^{p}-\bar{m}^{p}-\bar{M}^{p} \geq 0, \quad \bar{X}=\frac{1}{2} 1_{K}-\frac{1}{\bar{M}-\bar{m}}\left|\Phi(X)-\frac{\bar{M}+\bar{m}}{2} 1_{K}\right| \geq 0, \\
& \delta_{2}=d\left(2^{1-q}(\bar{n}+\bar{N})^{q}-\bar{n}^{q}-\bar{N}^{q}\right) \leq 0, \quad \bar{Y}=\frac{1}{2} 1_{K}-\frac{1}{\bar{N}-\bar{n}}\left|\Psi(Y)-\frac{\bar{N}+\bar{n}}{2} 1_{K}\right| \geq 0 .
\end{aligned}
$$

(ii) If $p, q \in[0,1] \cup[2, \infty), p^{2}-p \leq q^{2}-q$, and $\alpha=c^{p-2}\left(p^{2}-p+q^{2}-q\right) / 2$, then $f \in$ $\mathcal{K}_{1}^{c}([0, \infty))$. If

$$
C_{1}:=\frac{\alpha}{2}\left[\Phi\left(X^{2}\right)-\Phi(X)^{2}\right] \leq C_{2}:=\frac{\alpha}{2}\left[\Psi\left(Y^{2}\right)-\Psi(Y)^{2}\right],
$$

then (16) gives

$$
\Phi\left(X^{p}\right)-\Phi(X)^{p}+\delta_{1} \bar{X} \leq C_{1} \leq C_{2} \leq d \Psi\left(Y^{q}\right)-d \Psi(Y)^{q}+\delta_{2} \bar{Y}
$$

where

$$
\begin{aligned}
& \delta_{1}=2^{1-p}(\bar{m}+\bar{M})^{p}-\bar{m}^{p}-\bar{M}^{p}+\alpha(\bar{M}-\bar{m})^{2} / 4 \geq 0, \\
& \delta_{2}=d\left(2^{1-q}(\bar{n}+\bar{N})^{q}-\bar{n}^{q}-\bar{N}^{q}\right)+\alpha(\bar{N}-\bar{n})^{2} / 4 \leq 0,
\end{aligned}
$$

and $\bar{X}, \bar{Y} \geq 0$ as in the case (i). 
(iii) If $p, q \in(-\infty, 0] \cup[1,2], p^{2}-p \geq q^{2}-q$, and $\alpha=c^{p-2}\left(p^{2}-p+q^{2}-q\right) / 2$, then $f \in$ $\mathcal{K}_{2}^{c}([0, \infty))$. If $C_{1} \geq C_{2}(>0)$, then (16) gives

$$
\Phi\left(X^{p}\right)-\Phi(X)^{p}+\delta_{1} \bar{X} \geq C_{1} \geq C_{2} \geq d \Psi\left(Y^{q}\right)-d \Psi(Y)^{q}+\delta_{2} \bar{Y}
$$

where $\delta_{1} \geq 0, \delta_{2} \leq 0$, and $\bar{X}, \bar{Y} \geq 0$ as in the case (ii).

The first and the last inequality in (16) are obvious, so we omit them.

Levinson's operator inequality (7) holds with the weaker condition: $f \in \mathcal{K}_{1}^{c}(I)$ and with spectra conditions (see [3], Theorem 5). Next, applying Theorem 5 we obtain a refinement of this inequality. The proof is the same as for Corollary 2 and we omit the details.

Corollary 6 Let $\left(\Phi_{1}, \ldots, \Phi_{k_{1}}\right)$ be a unital $k_{1}$-tuple and $\left(\Psi_{1}, \ldots, \Psi_{k_{2}}\right)$ be a unital $k_{2}$-tuple of positive linear mappings $\Phi_{i}, \Psi_{j}: \mathcal{B}(H) \rightarrow \mathcal{B}(K)$. Let $\left(X_{1}, \ldots, X_{k_{1}}\right)$ be a $k_{1}$-tuple and $\left(Y_{1}, \ldots, Y_{k_{2}}\right)$ be a $k_{2}$-tuple of self-adjoint operators $X_{i}$ and $Y_{j} \in \mathcal{B}_{h}(H)$ with spectra contained in $\left[m_{i}, M_{i}\right]$ and $\left[n_{j}, N_{j}\right]$, respectively, such that

$$
\begin{aligned}
& a<m_{i} \leq M_{i} \leq c \leq n_{j} \leq N_{j}<b, \quad i=1, \ldots, k_{1}, j=1, \ldots, k_{2}, \\
& \left(m_{x}, M_{x}\right) \cap\left[m_{i}, M_{i}\right]=\varnothing, \quad i=1, \ldots, k_{1}, \quad\left(m_{y}, M_{y}\right) \cap\left[n_{j}, N_{j}\right]=\varnothing, \quad j=1, \ldots, k_{2}, \\
& m<M, \quad n<N
\end{aligned}
$$

where $m_{x}, M_{x}$ and $n_{y}, N_{y}$ are bounds of $X=\sum_{i=1}^{k_{1}} \Phi_{i}\left(X_{i}\right)$ and $Y=\sum_{i=1}^{k_{2}} \Psi_{i}\left(Y_{i}\right)$, respectively, and

$$
\begin{aligned}
& m:=\max \left\{M_{i} \mid M_{i} \leq m_{x}, i=1, \ldots, k_{1}\right\}, \quad M:=\min \left\{m_{i} \mid m_{i} \geq M_{x}, i=1, \ldots, k_{1}\right\}, \\
& n:=\max \left\{N_{i} \mid N_{i} \leq n_{y}, i=1, \ldots, k_{2}\right\}, \quad N:=\min \left\{n_{i} \mid n_{i} \geq N_{y}, i=1, \ldots, k_{2}\right\} .
\end{aligned}
$$

Iff $\in \mathcal{K}_{1}^{c}((a, b))$ and $D_{1} \leq D_{2}($ see $(8))$, then

$$
\begin{aligned}
& \sum_{i=1}^{k_{1}} \Phi_{i}\left(f\left(X_{i}\right)\right)-f\left(\sum_{i=1}^{k_{1}} \Phi_{i}\left(X_{i}\right)\right) \\
& \leq \sum_{i=1}^{k_{1}} \Phi_{i}\left(f\left(X_{i}\right)\right)-f\left(\sum_{i=1}^{k_{1}} \Phi_{i}\left(X_{i}\right)\right)+\delta_{1} \bar{X} \leq D_{1} \\
& \leq D_{2} \leq \sum_{i=1}^{k_{2}} \Psi_{i}\left(f\left(Y_{i}\right)\right)-f\left(\sum_{i=1}^{k_{2}} \Psi_{i}\left(Y_{i}\right)\right) \\
& \leq \sum_{i=1}^{k_{2}} \Psi_{i}\left(f\left(Y_{i}\right)\right)-f\left(\sum_{i=1}^{k_{2}} \Psi_{i}\left(Y_{i}\right)\right)+\delta_{2} \bar{Y}
\end{aligned}
$$

where $\delta_{1}=\delta_{f, \alpha}(\bar{m}, \bar{M}) \geq 0, \bar{X}=\bar{\Delta}_{X}(\bar{m}, \bar{M}) \geq 0$ for arbitrary numbers $\bar{m} \in\left[m, m_{x}\right], \bar{M} \in$ $\left[M_{x}, M\right], \bar{m}<\bar{M}$ and $\delta_{2}=\delta_{f, \alpha}(\bar{n}, \bar{N}) \leq 0, \bar{Y}=\bar{\Delta}_{Y}(\bar{n}, \bar{N}) \geq 0$ for arbitrary numbers $\bar{n} \in\left[n, n_{y}\right]$, $\bar{N} \in\left[N_{y}, N\right], \bar{n}<\bar{N}$.

But, if $f \in \mathcal{K}_{2}^{c}((a, b))$ and $D_{1} \geq D_{2}$ holds, then the reverse inequalities are valid in (19), with $\delta_{1} \leq 0$ and $\delta_{2} \geq 0$. 


\section{Refined converse of Levinson's operator inequality}

In this section we obtain a refined converse of Levison's operator inequality (15) given in Section 2.

For convenience, we introduce the abbreviation

$$
\widetilde{\Delta} \equiv \widetilde{\Delta}_{\Phi, B}(m, M):=\Phi\left(\frac{1}{2} 1_{H}-\frac{1}{M-m}\left|B-\frac{m+M}{2} 1_{H}\right|\right),
$$

where $B \in \mathcal{B}_{h}(H)$ is a self-adjoint operator, $\Phi$ is a normalized positive linear mapping and $m, M(m<M)$ are some scalars such that spectra $\operatorname{Sp}(X) \subseteq[m, M]$. Obviously, $\widetilde{\Delta} \geq 0$.

First, we give a refinement of (9) for two pairs of operators.

Theorem 7 Let $\Phi, \Psi: \mathcal{B}(H) \oplus \mathcal{B}(H) \rightarrow \mathcal{B}(K)$ be normalized mappings such that $\Phi\left(\operatorname{diag}\left(B_{1}, B_{2}\right)\right)=\Phi_{1}\left(B_{1}\right)+\Phi_{2}\left(B_{2}\right)$ and $\Psi\left(\operatorname{diag}\left(B_{1}, B_{2}\right)\right)=\Psi_{1}\left(B_{1}\right)+\Psi_{2}\left(B_{2}\right)$, where $\Phi_{1}$, $\Phi_{2}, \Psi_{1}, \Psi_{2}$ are positive linear mappings. Let $X=\operatorname{diag}\left(X_{1}, X_{2}\right), Y=\operatorname{diag}\left(Y_{1}, Y_{2}\right)$, where $X_{1}, X_{2}, Y_{1}, Y_{2} \in \mathcal{B}_{h}(H)$ are self-adjoint operators with spectra $\operatorname{Sp}\left(X_{1}\right), \operatorname{Sp}\left(X_{2}\right) \subseteq[m, M]$, $\operatorname{Sp}\left(Y_{1}\right), \operatorname{Sp}\left(Y_{2}\right) \subseteq[n, N]$, such that $a<m \leq M \leq c \leq n \leq N<b$. Let $m_{x}, M_{x}$ and $n_{y}, N_{y}$ be bounds of the operators $\Phi(X)$ and $\Psi(Y)$, respectively (see Figure 3). Iff $\in \mathcal{K}_{1}^{c}((a, b))$ and $C_{1} \geq C_{2}($ see (4)), then

$$
\begin{aligned}
& \Phi(f(X))-f(\Phi(X))+\beta_{1} 1_{K} \\
& \quad \geq \Phi(f(X))-f(\Phi(X))+\beta_{1} 1_{K}-\delta_{1} \widetilde{X} \geq C_{1} \\
& \quad \geq C_{2} \geq \Psi(f(Y))-f(\Psi(Y))+\beta_{2} 1_{K}-\delta_{2} \widetilde{Y} \geq \Psi(f(Y))-f(\Psi(Y))+\beta_{2} 1_{K},
\end{aligned}
$$

where $\beta_{1}, \beta_{2}$ are defined as in Theorem $3, \delta_{1}=\delta_{f, \alpha}(m, M) \geq 0, \widetilde{X}=\widetilde{\Delta}_{\Phi, X}(m, M) \geq 0, \delta_{2}=$ $\delta_{f, \alpha}(n, N) \leq 0$, and $\widetilde{Y}=\widetilde{\Delta}_{\Psi, Y}(n, N) \geq 0$.

If $\in \in \mathcal{K}_{2}^{c}((a, b))$ and $C_{1} \leq C_{2}$ holds, then the reverse inequalities are valid in (20), with $\delta_{1} \leq 0$ and $\delta_{2} \geq 0$ and $\beta_{1}$ and $\beta_{2}$ as in Theorem 3 in the dual case.

Proof We will give the proof for $f \in \mathcal{K}_{1}^{c}((a, b))$. Since $F(t)=f(t)-\frac{\alpha}{2} t^{2}$ is concave on $[m, c] \subset$ $(a, c]$ for some constant $\alpha$, the refined converse of Jensen's inequality for a concave function implies (see [14], Theorem 8)

$$
\begin{aligned}
& \Phi(F(X))-F(\Phi(X)) \geq \min _{m_{x} \leq t \leq M_{x}}\left\{f_{\alpha,[m, M]}^{\text {line }}(t)-f(t)-\frac{\alpha}{2} t^{2}\right\} 1_{K}-\widetilde{\widetilde{\delta}}_{1} \widetilde{X} \\
& \Rightarrow \quad \Phi(f(X))-\frac{\alpha}{2} \Phi\left(X^{2}\right)-f(\Phi(X))+\frac{\alpha}{2} \Phi(X)^{2}+\beta_{1} 1_{K}+\widetilde{\widetilde{\delta}}_{1} \widetilde{X} \geq 0 \\
& \Rightarrow \quad \Phi(f(X))-f(\Phi(X))+\beta_{1} 1_{K}-\widetilde{\delta}_{1} \widetilde{X} \geq C_{1},
\end{aligned}
$$

since $0 \geq \widetilde{\widetilde{\delta}}_{1}=F(m)+F(M)-2 F\left(\frac{m+M}{2}\right)=-\delta_{f, \alpha}(m, M)=-\widetilde{\delta}_{1}$ and

$$
\begin{aligned}
\widetilde{X} & =\frac{1}{2} 1_{K}-\frac{1}{M-m}\left\{\Phi_{1}\left(\left|X_{1}-\frac{m+M}{2} 1_{H}\right|\right)+\Phi_{2}\left(\left|X_{2}-\frac{m+M}{2} 1_{H}\right|\right)\right\} \\
& =\frac{1}{2} 1_{K}-\frac{1}{M-m} \Phi\left(\left|X-\frac{m+M}{2} 1_{H}\right|\right) \\
& =\Phi\left(\frac{1}{2} 1_{H}-\frac{1}{M-m}\left|X-\frac{m+M}{2} 1_{H}\right|\right)=\widetilde{\Delta}_{\Phi, X}(m, M) .
\end{aligned}
$$


Similarly, since $F$ is convex on $\left[c, N_{2}\right] \subset[c, b)$ for some constant $\alpha$, the refined converse of Jensen's inequality for a convex function implies (see [14], Theorem 8)

$$
\begin{aligned}
& \Psi(F(Y))-F(\Psi(Y)) \leq \max _{n_{y} \leq t \leq N_{y}}\left\{f_{\alpha,[n, N]}^{\text {line }}(t)-f(t)-\frac{\alpha}{2} t^{2}\right\}-\widetilde{\widetilde{\delta}}_{2} \widetilde{Y} \\
& \Rightarrow \quad \Psi(f(Y))-\frac{\alpha}{2} \Psi\left(Y^{2}\right)-f(\Psi(Y))+\frac{\alpha}{2} \Psi(Y)^{2}+\beta_{2} 1_{K}+\widetilde{\widetilde{\delta}}_{2} \widetilde{Y} \leq 0 \\
& \Rightarrow \quad C_{2} \geq \Psi(f(Y))-f(\Psi(Y))+\beta_{2} 1_{K}-\widetilde{\delta}_{2} \widetilde{Y}
\end{aligned}
$$

since $0 \leq \widetilde{\widetilde{\delta}}_{2}=F(n)+F(N)-2 F\left(\frac{n+N}{2}\right)=-\delta_{f, \alpha}(n, N)=-\widetilde{\delta}_{2}$ and

$$
\widetilde{Y}=\Psi\left(\frac{1}{2} 1_{H}-\frac{1}{N-n}\left|Y-\frac{n+N}{2} 1_{H}\right|\right)=\widetilde{\Delta}_{\Psi, Y}(n, N) .
$$

Combining inequalities (21) and (22) we obtain the desired inequality (20).

Example 3 Let $\Phi_{i}, \Psi_{i}, X_{i}, Y_{i}, i=1,2, m, M \geq 0, n, N \geq 0, \Phi, \Psi, X, Y, m_{x}, M_{x}, n_{y}, N_{y}$ be as in Theorem 7.

We will apply Theorem 7 putting $f(t)=t^{p}$ on $(0, c], f(t)=d t^{q}$ on $[c, \infty)$, where $c>0$ and $d=c^{p-q}$.

(i) If $p \in(-\infty, 0] \cup[1, \infty), q \in[0,1]$, and $\alpha=0$, then reverse of (20) gives

$$
\Phi\left(X^{p}\right)-\Phi(X)^{p}+\beta_{1}^{\circ} 1_{K}-\delta_{1} \widetilde{X} \leq 0 \leq d \Psi\left(Y^{q}\right)-d \Psi(Y)^{q}+\beta_{2}^{\circ} 1_{K}-\delta_{2} \widetilde{Y},
$$

where $\beta_{1}^{\circ}, \beta_{2}^{\circ}$ are as in Example 1(i), and

$$
\begin{aligned}
& \delta_{1}=2^{1-p}(m+M)^{p}-m^{p}-M^{p} \geq 0, \quad \tilde{X}=\frac{1}{2} 1_{K}-\frac{1}{M-m} \Phi\left(\left|X-\frac{M+m}{2} 1_{H}\right|\right), \\
& \delta_{2}=d\left(2^{1-q}(n+N)^{q} / 2-n^{q}-N^{q}\right) \leq 0, \quad \tilde{Y}=\frac{1}{2} 1_{K}-\frac{1}{N-n} \Psi\left(\left|Y-\frac{N+n}{2} 1_{H}\right|\right) .
\end{aligned}
$$

(ii) If $p, q \in(-\infty, 0] \cup[1,2], p^{2}-p \geq q^{2}-q$, and $\alpha=c^{p-2}\left(p^{2}-p+q^{2}-q\right) / 2$, then $f \in$ $\mathcal{K}_{2}^{c}([0, \infty))$. If

$$
(0<) \quad C_{1}:=\frac{\alpha}{2}\left[\Phi\left(X^{2}\right)-\Phi(X)^{2}\right] \leq C_{2}:=\frac{\alpha}{2}\left[\Psi\left(Y^{2}\right)-\Psi(Y)^{2}\right],
$$

then the reverse of (20) gives

$$
\Phi\left(X^{p}\right)-\Phi(X)^{p}+\beta_{1} 1_{K}-\delta_{1} \widetilde{X} \leq C_{1} \leq C_{2} \leq d \Psi\left(Y^{q}\right)-d \Psi(Y)^{q}+\beta_{2} 1_{K}-\delta_{2} \tilde{Y}
$$

where $\beta_{1}, \beta_{2}$ are as in Example 1(ii),

$$
\begin{aligned}
& \delta_{1}=2^{1-p}(m+M)^{p}-m^{p}-M^{p}+\alpha(M-m)^{2} / 4 \geq 0, \\
& \delta_{2}=d\left(2^{1-q}(n+N)^{q}-n^{q}-N^{q}\right)+\alpha(N-n)^{2} / 4 \leq 0
\end{aligned}
$$

and $\tilde{X}, \tilde{Y}$ are as in the case (i). 
(iii) If $p, q \in[0,1] \cup[2, \infty), p^{2}-p \leq q^{2}-q$, and $\alpha=c^{p-2}\left(p^{2}-p+q^{2}-q\right) / 2$, then $f \in$ $\mathcal{K}_{1}^{c}([0, \infty))$. If $C_{1} \geq C_{2}$, then (20) gives

$$
\Phi\left(X^{p}\right)-\Phi(X)^{p}+\gamma_{1} 1_{K}-\delta_{1} \tilde{X} \geq C_{1} \geq C_{2} \geq d \Psi\left(Y^{q}\right)-d \Psi(Y)^{q}+\gamma_{2} 1_{K}-\delta_{2} \tilde{Y}
$$

where $\gamma_{1} \geq 0$ is defined similar to $\beta_{1}$ with max instead of min and $\gamma_{2} \leq 0$ is defined similar to $\beta_{2}$ with min instead of $\max$, and $\delta_{1} \leq 0, \delta_{2} \geq 0, \tilde{X}, \tilde{Y}$ are as in the case (ii).

The first and the last inequality in (20) are obvious, so we omit them.

Remark 4 Let the assumptions of Theorem 5 be satisfied. If $f \in \dot{\mathcal{K}}_{1}^{c}\left(\left[m_{1}, N_{2}\right]\right)$ and $C_{1} \geq C_{2}$, we obtain the following extension of (16):

$$
\begin{aligned}
C_{1}+\beta_{1} 1_{K} & \geq \Phi(f(X))-f(\Phi(X))+\beta_{1} 1_{K} \\
& \geq \Phi(f(X))-f(\Phi(X))+\beta_{1} 1_{K}-\delta_{1} \bar{X} \geq C_{1} \\
& \geq C_{2} \geq \Psi(f(Y))-f(\Psi(Y))+\beta_{2} 1_{K}-\delta_{2} \bar{Y} \\
& \geq \Psi(f(Y))-f(\Psi(Y))+\beta_{2} 1_{K} \geq C_{2}+\beta_{2} 1_{K} .
\end{aligned}
$$

But, if $f \in \dot{\mathcal{K}}_{2}^{c}((a, b))$ and $C_{1} \leq C_{2}$, then the reverse inequalities are valid in (23).

Applying Theorem 7 we obtain a refinement of (15). We omit the proof.

Corollary 8 Let $\left(\Phi_{1}, \ldots, \Phi_{k_{1}}\right)$ be a unital $k_{1}$-tuple and $\left(\Psi_{1}, \ldots, \Psi_{k_{2}}\right)$ be a unital $k_{2}$-tuple of positive linear mappings $\Phi_{i}, \Psi_{j}: \mathcal{B}(H) \rightarrow \mathcal{B}(K)$. Let $\left(X_{1}, \ldots, X_{k_{1}}\right)$ be a $k_{1}$-tuple and $\left(Y_{1}, \ldots, Y_{k_{2}}\right)$ be a $k_{2}$-tuple of self-adjoint operators $X_{i}$ and $Y_{j} \in \mathcal{B}_{h}(H)$ with spectra contained in $[m, M]$ and $[n, N]$, respectively, such that $a<m \leq M \leq c \leq n \leq N<b$. Let $m_{x}, M_{x}$ and $n_{y}, N_{y}$ be bounds of $X=\sum_{i=1}^{k_{1}} \Phi_{i}\left(X_{i}\right)$ and $Y=\sum_{i=1}^{k_{2}} \Psi_{i}\left(Y_{i}\right)$, respectively.

Iff $\in \mathcal{K}_{1}^{c}((a, b))$ and $D_{1} \geq D_{2}$ (see (8)), then

$$
\begin{aligned}
& \sum_{i=1}^{k_{1}} \Phi_{i}\left(f\left(X_{i}\right)\right)-f\left(\sum_{i=1}^{k_{1}} \Phi_{i}\left(X_{i}\right)\right)+\beta_{1} 1_{K} \\
& \geq \sum_{i=1}^{k_{1}} \Phi_{i}\left(f\left(X_{i}\right)\right)-f\left(\sum_{i=1}^{k_{1}} \Phi_{i}\left(X_{i}\right)\right)-\delta_{1} \widetilde{X}+\beta_{1} 1_{K} \geq D_{1} \\
& \geq D_{2} \geq \sum_{i=1}^{k_{2}} \Psi_{i}\left(f\left(Y_{i}\right)\right)-f\left(\sum_{i=1}^{k_{2}} \Psi_{i}\left(Y_{i}\right)\right)+\beta_{2} 1_{K}-\delta_{2} \widetilde{Y} \\
& \geq \sum_{i=1}^{k_{2}} \Psi_{i}\left(f\left(Y_{i}\right)\right)-f\left(\sum_{i=1}^{k_{2}} \Psi_{i}\left(Y_{i}\right)\right)+\beta_{2} 1_{K},
\end{aligned}
$$

where $\beta_{1}$ and $\beta_{2}$ are defined as in Theorem $3, \delta_{1}=\delta_{f, \alpha}(m, M) \geq 0, \widetilde{X}=\sum_{i=1}^{k_{1}} \widetilde{\Delta}_{\Phi_{i}, X_{i}}(m, M) \geq$ $0, \delta_{2}=\delta_{f, \alpha}(n, N) \leq 0$, and $\widetilde{Y}=\sum_{i=1}^{k_{2}} \widetilde{\Delta}_{\Psi_{i}, y_{i}}(n, N) \geq 0$.

Iff $\in \mathcal{K}_{2}^{c}((a, b))$ and $D_{1} \leq D_{2}$ holds, then the reverse inequalities are valid in (24), with $\delta_{1} \leq 0$ and $\delta_{2} \geq 0$ and $\beta_{1}$ and $\beta_{2}$ as in Theorem 3 in the dual case. 
Competing interests

The authors declare that they have no competing interests.

\section{Authors' contributions}

All authors contributed equally and significantly in writing this paper. All authors read and approved the final manuscript.

\section{Author details}

${ }^{1}$ Faculty of Mechanical Engineering and Naval Architecture, University of Zagreb, Ivana Lučića 5, Zagreb, 10000, Croatia.

${ }^{2}$ Faculty of Textile Technology, University of Zagreb, Prilaz baruna Filipovića 30, Zagreb, 10000, Croatia.

\section{Acknowledgements}

This work has been supported in part by the Croatian Science Foundation under the project 5435

Received: 12 November 2014 Accepted: 20 March 2015 Published online: 09 April 2015

\section{References}

1. Furuta, T, Mićić Hot, J, Pečarić, J, Seo, Y: Mond-Pečarić Method in Operator Inequalities. Monographs in Inequalities, vol. 1. Element, Zagreb (2005)

2. Fujii, T, Mićić Hot, J, Pečarić, J, Seo, Y: Recent Developments of Mond-Pečarić Method in Operator Inequalities. Monographs in Inequalities, vol. 4. Element, Zagreb (2012)

3. Mićić, J, Pečarić, J, Praljak, M: Levinson's inequality for Hilbert space operators. J. Math. Inequal. (acceptted)

4. Levinson, N: Generalization of an inequality of Ky Fan. J. Math. Anal. Appl. 8, 133-134 (1964)

5. Popoviciu, T: Sur une inégalité de N. Levinson. Mathematica 6, 301-306 (1964)

6. Bullen, PS: An inequality of N. Levinson. Publ. Elektroteh. Fak. Univ. Beogr., Ser. Mat. Fiz. 412-460, 109-112 (1973)

7. Mercer, AM: Short proof of Jensen's and Levinson's inequalities. Math. Gaz. 94, 492-495 (2010)

8. Witkowski, A: On Levinson's inequality. RGMIA Res. Rep. Collect. 15, 68 (2012)

9. Witkowski, A: On Levinson's inequality. Ann. Univ. Paedagog. Crac. Stud. Math. 12, 59-67 (2013)

10. Baloch, IA, Pečarić, J, Praljak, M: Generalization of Levinson's inequality. J. Math. Inequal. (acceptted)

11. Pečarić, J, Praljak, M, Witkowski, A: Generalized Levinson's inequality and exponential convexity. Opusc. Math. 35 , 397-410 (2015)

12. Mićić, J, Pavić, Z, Pečarić, J: Some better bounds in converses of the Jensen operator inequality. Oper. Matrices 6 , 589-605 (2012)

13. Mićić, J, Pečarić, J, Perić, J: Refined Jensen's operator inequality with condition on spectra. Oper. Matrices 7, 293-308 (2013)

14. Mićić, J, Pečarić, J, Perić, J: Refined converses of Jensen's inequality for operators. J. Inequal. Appl. 2013, 353 (2013)

\section{Submit your manuscript to a SpringerOpen ${ }^{\circ}$ journal and benefit from:}

- Convenient online submission

Rigorous peer review

- Immediate publication on acceptance

- Open access: articles freely available online

- High visibility within the field

- Retaining the copyright to your article 